\title{
BENCHMARKING FOR PROCESS CONTROL WITH APPLICATIONS IN THE HOT STRIP FINISHING STEEL MILL
}

\author{
David Greenwood, Michael A. Johnson, Michael J. Grimble \\ Industrial Control Centre, University of Strathclyde, Glasgow, Scotland, U. K. \\ Email: m.johnson@eee.strath.ac.uk, Fax: +44 (0)141 5484203
}

Keywords benchmarking concepts, economic process benchmarking, technical process benchmarking, control loop benchmarking, process control

\begin{abstract}
This paper describes how new benchmarking concepts can be applied to different aspects of process control performance assessment. Three general levels of benchmarking concepts for process and industrial control applications are introduced and defined: economic process, technical process and control loop benchmarking. The concepts of technical process and control loop benchmarking are demonstrated using the finishing mill in the hot strip steel rolling process. The example for technical process benchmarking is that of the control of load distribution on a hotstrip finishing mill. The example for control loop benchmarking is the multivariable control of the interstand looper in the finishing mill.
\end{abstract}

\section{INTRODUCTION}

In industrial applications of control one of the most common business questions to arise is the one of whether a new (advanced) control design will bring any benefits. This question is usually coupled to one about whether additional investment is needed to bring any improvements in process operation, throughput, or process quality. Over the last fifteen years a number of concepts and tools have been evolving concerned with answering this type of question. The emergence of this industrial controller assessment paradigm has shadowed the growth of the culture of business process benchmarking found in commercial organisations. The now well-established technique of business process benchmarking was first outlined by the Rank Xerox company in the late 1980's and eventually led to a formal ten step procedure (Cross and Iqbal, 1995). Since then business process benchmarking has been explored by various authors to produce several similar formal procedures which are now viewed as an important tools in a Total Quality Management (TQM) toolbox (Anderson and Petterson, 1995). In this paper consideration is given to defining and using analogous technical process benchmarking concepts in addition to the usual control loop benchmarking as a contribution to the development of a complete assessment philosophy for process control performance.

\section{Layout of the paper}

In section 2, the fundamental principles and classification for the benchmarking method for use in process control are established. Three general levels of benchmarking concepts for process and industrial control applications are defined: economic process, technical process and control loop benchmarking. In section 3, the applications of technical process benchmarking is demonstrated through the control of load distribution on a hotstrip finishing mill. This achieved through the application of GPC. The original feature of this presentation is the construction of a novel benchmarking cost function. In Section 4, the application of LQG restricted structure controller design for the control of interstand looper in the finishing mill is presented as an example of control loop performance assessment. This example is used to show the performance benefit obtained by introducing different levels of structural complexity into the multivariable controller. Conclusions and References close the paper.

\section{CONTROL BENCHMARKING}

\subsection{The benchmarking fundamentals}

The benchmarking method relies on using a performance metric which has the following features:

(i) The performance metric should be technically meaningful for the process being assessed.

(ii) The performance metric should be computable from straightforward process data (online or offline).

(iii) The performance metric should preferably be amenable to optimisation to enable the full achievable optimised performance to be computed.

In control assessments, an optimisation framework is used to provide a conceptual and quantitative basis for benchmarking studies. A fundamental principle in optimisation which provides the conceptual basis is given by the following lemma.

\section{Lemma 1 Benchmarking Fundamentals}

Let $J: V \rightarrow \mathfrak{R}^{+}$represent a benchmark performance index defined on vector space $V$, and with $\mathfrak{R}^{+}=[0, \infty]$. Let $C_{1}: V \rightarrow V_{1}$ and $C_{2}: V \rightarrow V_{2}$ represent two constraint 
relationships. Define three optimisation problems and solutions,

$J\left(x^{0}\right)=\left\{\begin{array}{c}\min J(x) \\ x \in V\end{array}\right\}, J\left(x^{*}\right)=\left\{\begin{array}{c}\min J(x) \\ x \in V \\ C_{1}(x) \leq 0\end{array}\right\}, J\left(x^{* *}\right)=\left\{\begin{array}{c}\min J(x) \\ x \in V \\ C_{1}(x) \leq 0 \\ C_{2}(x) \leq 0\end{array}\right\}$

then $J\left(x^{\circ}\right) \leq J\left(x^{*}\right) \leq J\left(x^{* *}\right)$

Proof: By contradiction.

The principle of nested optimisation enables different interpretations to be established for benchmarking assessment. As an example, the cost $J\left(x^{\circ}\right)$ represents the optimal performance, which can be obtained with no constraints operating. Constraint set $C_{1}($.) might be related to control design whilst additional constraint set $C_{2}$ (.) might be related to implementation. Thus the following differences can be considered and given diagnostics interpretations,

Cost difference $\Delta J_{1}=J\left(x^{*}\right)-J\left(x^{\circ}\right)$ - Degradation in performance due to design constraints.

Cost difference $\Delta J_{2}=J\left(x^{* *}\right)-J\left(x^{*}\right)$ - Additional loss

in performance due to a particular implementation. Another way of using the nested costs of (1) is as the set of inequalities,

$1 \leq \frac{J\left(x^{*}\right)}{J\left(x^{0}\right)} \leq \frac{J\left(x^{* *}\right)}{J\left(x^{0}\right)}$

Thus the closeness of the cost ratios can be given a diagnostic interpretation and nested optimisation meets the requirement of Petersen (1995) for a correlation between achievable performance and the cause of the loss of performance.

\subsection{Benchmarking in process control}

Several procedures for control loop benchmarking have emerged and it is possible to place these methods in a coherent conceptual framework. For this the standard process control hierarchy of three process control levels is used(Johnson and Grimble, 1999):

Top Level - process units globally co-ordinated by automation and operator systems

Unit Level - complex process units operated to fit seamlessly with the global process line co-ordination

Subunit Level - process regulator structure operates autonomously

Using this process hierarchy, the three general classes of benchmarking methods emerge.

\section{Economic process benchmarking}

Economic process benchmarking is quite similar to business process benchmarking. To give a flavour of this methodology it is useful to cite the typical economic process benchmarking categories: customer service levels, asset reliability, operational excellence, personnel metrics, safety, healthy and environment (Ahmad and Benson, 1999). Thus, economic process benchmarking is taking place at the business and technical plant interface that occurs at the top of the process control hierarchy.

\section{Technical process benchmarking}

This topic is the least well known of the three control benchmarking activities, yet it is believed to have significant potential in the set of techniques. To manage a layered hierarchical or large-scale or complex industrial processes the system designer usually imposes a structured design solution. This structure is the way that the designer expects the system to work. Typically this will involve concepts like decoupled operation, or sequences of structured values for the internal variables of the process. These are the designer's way of ensuring that the process actually works and remains operationally stable whilst achieving the desired quality outputs. These structural measures can be used as the basis of technical process benchmarks. Since this operational structure is usually designed in at the unit level and at the level of interaction between units, technical process benchmarks occur in the middle of the process hierarchy. Examples of the use of these ideas were given by Calligaris and Johnson (1999, 2000) for the voltage control of the national power transmission systems of Italy and Spain. In this paper, an example from the operation of a hot strip rolling steel mill as found in the steel industry is given. A more detailed presentation has been given by Greenwood (2003). An interesting potential of process technical benchmarking is to incorporate explicit control action in the process supervisory layers to improve benchmark attainment during real-time operation. This category of techniques is given the name technical process benchmarking to mirror the analogous term business process benchmarking as used in the commerce.

\section{Control loop benchmarking}

The philosophical framework established for process control benchmarking straightforwardly subsumes the more well known work on control loop benchmarking which occurs at the regulator level of the process control hierarchy. The work of Harris and colleagues (Harris, 1989; Desborough and Harris, 1990) initiated control loop benchmarking using a minimum variance benchmark. More recent contributions to the field have included work by Huang, Shah and colleagues (Huang et al, 1997; Huang and Shah, 1999); Thornhill et al (1999) and Grimble (2002). All of these contributions used an optimal methodology that was underpinned by the benchmark lemma. By way of contrast, Huang and Jeng (2001) have used classical control measures to construct a benchmark procedure for process control loop benchmarking. 
Using Table 1, these benchmark methods are contextualised using the standard process hierarchy.

\begin{tabular}{|c|c|c|}
\hline $\begin{array}{l}\text { Level } \\
4\end{array}$ & $\begin{array}{l}\text { Load Management } \\
\text { Process Line Interfaces }\end{array}$ & $\begin{array}{l}\text { Economic process } \\
\text { benchmarking }\end{array}$ \\
\hline $\begin{array}{l}\text { Level } \\
3\end{array}$ & $\begin{array}{l}\text { Set-point Optimisation } \\
\text { Top Level Process Unit } \\
\text { Interface }\end{array}$ & \multirow[t]{2}{*}{$\begin{array}{l}\text { Technical process } \\
\text { benchmarking }\end{array}$} \\
\hline $\begin{array}{l}\text { Level } \\
2\end{array}$ & $\begin{array}{l}\text { Dynamic Set-point } \\
\text { Changeover } \\
\text { Automated Unit Level } \\
\text { Procedures }\end{array}$ & \\
\hline $\begin{array}{l}\text { Level } \\
1\end{array}$ & $\begin{array}{l}\text { Regulator Loop Control } \\
\text { Low Level Control } \\
\text { Structure and Controllers }\end{array}$ & $\begin{array}{l}\text { Control loop } \\
\text { benchmarking }\end{array}$ \\
\hline $\begin{array}{l}\text { Level } \\
0\end{array}$ & \multicolumn{2}{|c|}{$\begin{array}{l}\text { Process } \\
\text { Actuators, Process Equipment, Sensors }\end{array}$} \\
\hline
\end{tabular}

\subsection{The hot strip finishing mill application}

The hotstrip mill is the first stage in the manufacture of steel strip. The steel strip produced is typically used in consumer white-goods and car bodies. The hotstrip mill comprises a sequence of strip processing units. These begin with casting shop and the reheating furnaces and then move on to the preliminary rolling in the roughing mills. Steel ingots enter the hotstrip mill process at temperatures of around $1000^{\circ} \mathrm{C}$ and finish at the steel strip coiling section with the rolled strip at $350^{\circ} \mathrm{C}$. The major dimensional transformation to steel strip takes place in the hotstrip finishing mill. It is from this hotstrip finishing mill process that the two examples for benchmarking studies are taken. The output product of the hot strip mill is fed to a second rolling process known as the cold rolling mill. In this mill, as the name suggests, the rolling of strip occurs at ambient temperature.

\section{TECHNICAL PROCESS BENCHMARKING AND THE HOT STRIP MILL APPLICATION}

Technical process performance metrics relate to how well the process is operating. Thus the assessment takes place at the co-ordination level of the process. The important task is that of constructing metrics that meaningfully describe how well the process co-ordination is operating. For example, it may be that decoupled control of subunits is desired and the technical process benchmark would measure the decoupling achieved against an optimal level of decoupling. Clearly detailed process knowledge is used in the definition of the metric. The arguments used to create the technical process benchmark for hotstrip finishing mill are given next.

\subsection{Load balancing across the mill}

Before the rolling process commences, a set-up algorithm calculates appropriate values for the control loops on the plant. These values include a set of stand roll force set points that will produce a desired gauge reduction schedule across the finishing mill. This is to prevent all the gauge reduction occurring at one or two stands. This is both for metallurgical reasons and to prevent the drive motors from doing excessive work and surpassing their power ratings. Usually these reduction references are not changed by the automatic systems during rolling, only by operators if they see need for adjustment.

Load balancing is based on initial values of forces that a set-up program calculates for the $i$ th stand. It is the ratio of these forces that form the reference for the load distribution control problem.

$$
\lambda_{\text {setup }}^{i}=P_{\text {setup }}^{i} / \sum_{f=1}^{n} P_{\text {setup }}^{f} \quad i=1, \ldots n
$$

The set-up load ratios are defined over the period of the coil processing time,

$$
\lambda_{\text {setup }}(t) \in \mathfrak{R}^{n}, \quad 0 \leq t \leq T_{\text {coil }}
$$

For the purposes of benchmarking, the measured load ratios from measured forces on the mill during slab processing,

$$
\lambda_{m}^{i}=P_{m}^{i} / \sum_{f=1}^{n} P_{m}^{f}\left(t+\tau_{i f}\right) \quad i=1, \ldots n
$$

This ensures that the forces applied to a segment of strip as it passes through the mill are used to determine how well load distribution occurs. The difference can be seen if equation (5) is compared with instantaneous (or across the mill) load distribution measurement:

$$
\lambda_{m}^{i}=P_{m}^{i} / \sum_{f=1}^{n} P_{m}^{f} \quad i=1, \ldots n
$$

To control the plant for load distribution, a co-ordinated control structure is used to add trims to the exit gauge references. Due to the causal nature of the process, only downstream stand actions are considered in the control action. This is reflected in the way the reference ratios, those used by the controller and not the benchmark, are calculated,

$$
\lambda_{\text {ref }}^{i}=P_{\text {setup }}^{i} / \sum_{f=i}^{n} P_{\text {setup }}^{f} \quad i=1, \ldots n-1
$$

The control action must now minimise error to these references with the prediction of forces at the present stand and future downstream stands.

$$
\lambda_{\text {pred }}^{i}=\hat{P}_{\text {pred }}^{i} / \sum_{f=i}^{n} \hat{P}_{\text {pred }}^{f}\left(t+\tau_{\text {if }}\right) \quad i=1, \ldots n-1
$$


The feed forward control action needed to solve this control problem led to the use of Generalised Predictive Control. One task of the controller is to predict future load ratios from current data. It achieves this by predicting future forces based on the states of the system at that time from the simplified models. The prediction horizon for the controller is the maximum time the strip is in the mill, equivalent of the time taken to pass from stand 1 to 7 . The aim of the GPC cost equation is to determine the predicted future forces in order to calculate the estimated load ratios.

The standard cost function is given as,

$$
J=(R-Y)^{T} Q_{e}(R-Y)+U^{T} Q_{u} U
$$

where the references, $R$ and outputs, $Y$ represent the set-up load ratios, $\Lambda_{\text {setup }}$, and measured load ratios, $\Lambda_{m}$, respectively, and the weightings are user defined diagonal sparse matrices.

\subsection{Benchmarking the results}

The theoretical minimum cost is often unachievable due to design and implementation considerations, or sometimes undesirable due to the control actions produced. An optimal controller produced adopting the constraints placed on its design can provide a realistic minimum achievable cost. This optimal controller becomes the benchmark on which to compare other controller's responses.

The values of the cost function, (9), can be obtained for:

(a) existing controls or new designs, $J\left(\lambda_{\text {control }}\right)$

(b) an optimised cost function value obtained using an optimal control scheme, $J\left(\lambda_{\text {opt }}\right)$

The two cost values satisfy:

$$
0 \leq J\left(\lambda_{\text {opt }}\right) \leq J\left(\lambda_{\text {control }}\right)
$$

The ratio of these costs can be used to calculate a benchmark index of performance for the setup as,

$$
1 \leq B_{\text {setup }}=J\left(\lambda_{\text {control }}\right) / J\left(\lambda_{\text {opt }}\right)
$$

For values of the benchmark, $B_{\text {setup }}$, close to unity the performance of the control system is near optimal. But values of the benchmark, $B_{\text {setup }}$, much greater than 1 indicates that control performance is far from optimal and that there is room for improvement.

\section{CONTROL LOOP BENCHMARKING AND THE HOT STRIP MILL APPLICATION}

\subsection{Interstand looper control loop benchmarking}

The hotstrip finishing mill is a tandem mill of six or seven stands. Each roll-stand being a four-high roll stand. The outer two rolls are large diameter back-up rolls which provide stiffness for the two inner work rolls. The smaller diameter work rolls are in contact with the hot steel strip. The purpose of the roll stands is to effect a gauge (thickness) reduction in the strip as it travels through the mill. Between each pair of stands is a looper arm as shown in Figure 1. The looper arm can be raised or lowered to maintain the steel strip in tension as it is rolled. The looper arm angle is about $15^{\circ}$, but this angle varies in reaction to tension disturbances and weight changes. The control of looper angle and strip tension is a multivariable problem with considerable process interaction.

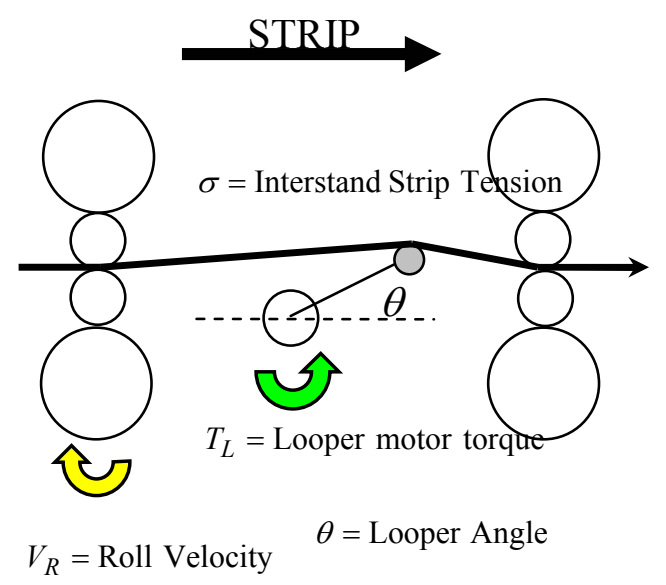

Figure 1 Interstand looper system

\subsection{Multivariable Controller Assessment}

A multivariable controller assessment procedure should have three components:

(a) An unconstrained optimal benchmark index and a straightforward design setup methodology.

(b) A method to compute the benchmark for different industrial control structures but with optimised parameters.

(c) A value of the benchmark for the implemented industrial controller.

The major assumption of the multivariable benchmarking procedure used with the multivariable interstand looper control problem was that the desired control design for an industrial control system can be captured adequately by LQG optimal control theory. The LQG cost function benchmark, $J_{L Q G}$ is used in a form appropriate for use with polynomial system methods and analysis (Grimble and Johnson, 1988). Thus, the steady state stochastic LQG quadratic cost function is given by,

$$
\begin{aligned}
& J_{L Q G}= \\
& \quad \frac{1}{2 \pi j} \int_{D}\left\{\operatorname{trace}\left\{Q_{c}(s) \Phi_{e e}(s)\right\}+\operatorname{trace}\left\{R_{c}(s) \Phi_{u u}(s)\right\}\right\} d s
\end{aligned}
$$


Where $\Phi_{e e}(s), \Phi_{u u}(s)$ are rational matrix spectral density transfer functions for the error $e(s)$ and the control $u(s)$. The integration is around the usual $\mathrm{D}$ contour. The multivariable dynamic weights are denoted $Q_{c}(s)$ and $R_{c}(s)$; these are used to shape the response produced by the controller.

A simple procedure to setup the LQG controller design parameters is presented by Greenwood et al (2003). The method to compute the benchmark for different industrial control structures but with optimised parameters is that of the optimal restricted structure multivariable controller. New results are needed and these are presented by Greenwood et al (2003). The new results are non-trivial extensions of existing scalar system optimal LQG benchmark results (Grimble 2000). Finally, the adoption of LQG cost function as a benchmark ensures that the computation of the benchmark for the implemented industrial controller is a straightforward operation.

A restricted structure multivariable controller has an internal structure with two components; a matrix structure where it is decided which controller elements are zero and which will have a controller element and a second structure where the actual form of the controller element itself will be determined(for example, a P, PI or a PID controller element. For simplicity, denote the internal restricted structure of $K_{R S}$ by the symbol, $S_{l}$ and denote the corresponding controller, $K_{R S}\left(S_{l}\right)$, then the restricted structure optimisation problem can be given as,

$$
\min _{\text {w.r.t. } K_{R S}\left(S_{l}\right)} J_{R S}\left(S_{l}\right)
$$

and $K_{R S}\left(S_{l}\right)$ closed loop stabilising

If the optimal value of the restricted structure optimisation problem for structure $S_{l}$ is denoted $J_{R S}^{o p t}\left(S_{i}\right)$, then the optimal cost function ordering for (say) $S_{l 1} \subset S_{l 2} \subset S_{l 3}$ will be,

$$
\begin{aligned}
J_{L Q G}^{o p t} \leq J_{L Q G}^{o p t}+J_{R S}^{o p t}\left(S_{l 3}\right) \leq J_{L Q G}^{o p t} & +J_{R S}^{o p t}\left(S_{l 2}\right) \\
& \leq J_{L Q G}^{o p t}+J_{R S}^{o p t}\left(S_{l 1}\right)
\end{aligned}
$$

Alternatively this inequality sequence can be used to benchmark the performance of restricted structure controllers solutions through the set of inequalities like,

$$
0 \leq \frac{J_{R S}^{o p t}\left(S_{l 3}\right)}{J_{L Q G}^{o p t}} \leq \frac{J_{R S}^{o p t}\left(S_{l 2}\right)}{J_{L Q G}^{o p t}} \leq \frac{J_{R S}^{o p t}\left(S_{l 1}\right)}{J_{L Q G}^{o p t}}
$$

$1 \leq \frac{J_{L Q G}^{o p t}+J_{R S}^{o p t}\left(S_{l 3}\right)}{J_{L Q G}^{o p t}} \leq \frac{J_{L Q G}^{o p t}+J_{R S}^{o p t}\left(S_{l 2}\right)}{J_{L Q G}^{o p t}} \leq \frac{J_{L Q G}^{o p t}+J_{R S}^{o p t}\left(S_{l 1}\right)}{J_{L Q G}^{o p t}}$

These inequalities form the basis of the benchmarking results and enable both a comparison of different controller structures but also the type of controller within a given control structure.

A complete set of the results produced by the various methods and controller structures for the hotstrip looper control loop assessment can be found in Table 2. The equations (14) and (15b) can be used to track interesting conclusions from these optimal cost figures.

\subsection{CONCLUSIONS}

The concepts of benchmarking were surveyed and three general levels of benchmarking concepts for process and industrial control applications were identified. These were economic control performance, a new concept of technical process benchmarking and the conventional control loop benchmarking. Technical process performance benchmarking was seen to be analogous to the concept of business process benchmarking which is widely used in commercial fields. The control problems of the finishing mill in a hot strip steel rolling mill were successfully used to test and illustrate concepts of technical process benchmarking and conventional control loop benchmarking.

\section{Acknowledgements}

The authors thank the UKs EPSRC for support under grants GR/L98237 and (GR/R04683-01).

\section{REFERENCES}

Ahmed, M. and R. Benson, 1999, Benchmarking in the process industries, 155pp., ISBN 085295411 5, Institution of Chemical Engineers, Rugby, UK.

Anderson B. and P-G Pettersen, 1996, The Benchmarking Handbook, Chapman and Hall, ISBN 0-412-73520-2.

Calligaris M. and M. A. Johnson, 1999, Power System Studies and New Benchmarking Concepts, Proceedings of the IASTED ICCA , Banff, Canada, ISBN 0-88986-253-2, pp. 211-216

Calligaris M. and M. A. Johnson, 2000, Benchmarking for Hierarchical Voltage Control, IFAC Symposium on Power Plants and Power System Control 2000, Brussels, Belgium.

Cross, R. and A. Iqbal, 1995, The Rank Xerox Experience : Benchmarking Ten Years On, Chapter 1, pps. 3-10, Benchmarking : Theory and Practice, Editor A. Rolstadas, Chapman and Hall, IBSN 0-412-62680-2.

Desborough, L. D. and T. J. Harris, 1992, Performance 
assessment measures for univariate feedback control, Can. J. Chem. Engrg., Vol 70, 1186-1197

Greenwood D., M. A. Johnson, and M. J. Grimble, 2003, Multivariable LQG optimal control benchmarking and restricted structure controller tuning, ECC 2003

Greenwood D., 2003, PhD Thesis, Industrial Control Centre, University of Strathclyde, Glasgow, Scotland, UK.

Grimble, M. J. 2000, Restricted Structure LQG Optimal Control for Continuous Systems, IEE Proc. CTA, Vol 147, No 2, (185195), March

Grimble, M. J., 2002, Controller performance benchmarking and tuning using generalised minimum variance, Automatica, Vol 38, No 2, (2111-2120)

Grimble M. J. and M. A. Johnson, 1988, Optimal Control and Stochastic Estimation, Volume 1 \& 2, John Wiley and Sons, Chichester, UK.

Harris T.J., 1989, Assessment of Closed-Loop Performance, Canadian J. Chem. Engrg. Vol 67, (856-861).

Huang B and S. L. Shah, 1999, Performance Assessment of Control Loops, 255pp, ISBN 1-85233-639-0, 1999
Huang B., S.L., Shah and E.K. Kwok, 1997, Good, Bad or Optimal? Performance Assessment of Multivariable Processes, Automatica, Vol. 33, No.6, (1175-1183).

Huang, H.-P., and J.-C. Jeng, 2001, Assessment and Monitoring of Control Performance for Single Loop Systems, ind. Eng. Chem.. Res., Vol 41, (1297-1309).

Johnson M.A. and Grimble, M.J., 1999, Industrial Process Control, in Perspective in Control: New Concepts and Applications, Editor: T. Samad, IEEE Press, USA, ISBN 0-78035356, October.

Pettersen P.G., 1995, Benchmarking for Implementing a New Product Strategy, Chapter 14, pps. 120-125, In Benchmarking Theory and Practice, Editor A. Rolstadad, Chapman and Hall, ISBN 0-412-62680-2.

Thornhill, N. F., M. Oettinger and P. Fedenczuk, 1999, Refinery Wide Control Loop Performance Assessment, Journal Process Control, Vol 9, 109-124.

\begin{tabular}{|c|c|c|c|}
\hline \multicolumn{4}{|c|}{ Table 2 Hotstrip Finishing Mill Looper Assessment } \\
\hline Control Structure & $\begin{array}{l}\text { No. Controller } \\
\text { Parameters, } n_{c}\end{array}$ & $\begin{array}{c}\text { Cost Function } \\
\text { Value }\end{array}$ & CPI \\
\hline \multicolumn{4}{|l|}{ Decentralised Structure ZN Tuning } \\
\hline PI $(1,1) \mathrm{PI}(2,2)$ & 4 & 50018.35 & 53.36167 \\
\hline $\operatorname{PID}(1,1) \operatorname{PID}(2,2)$ & 5 & 37364.35 & 39.86185 \\
\hline \multicolumn{4}{|l|}{ Decentralised Restricted Structure } \\
\hline PI(1,1) PI(2,2) & 4 & 1987.746 & 2.120611 \\
\hline $\operatorname{PID}(1,1) \mathrm{PI}(2,2)$ & 5 & 1988.446 & 2.121358 \\
\hline PI(1,1) PID $(2,2)$ & 5 & 1970.246 & 2.101941 \\
\hline $\operatorname{PID}(1,1) \operatorname{PID}(2,2)$ & 6 & 1969.846 & 2.101514 \\
\hline PI $(1,2) \operatorname{PI}(2,1)$ & 4 & 1175.139 & 1.253688 \\
\hline \multicolumn{4}{|l|}{ Limited Interactive Restricted Structure } \\
\hline PI(1,1) PI(2,1) PI(2,2) & 6 & 1927.816 & 2.056675 \\
\hline PI(1,1) PI(2,1) PID(2,2) & 7 & 1905.544 & 2.032914 \\
\hline $\operatorname{PID}(1,1) \operatorname{PID}(2,1) \operatorname{PID}(2,2)$ & 9 & 1905.155 & 2.032499 \\
\hline $\mathrm{PI}(1,1) \mathrm{PI}(1,2) \mathrm{PI}(2,2)$ & 6 & 1291.538 & 1.377867 \\
\hline PI(1,1) PI(1,2) PID(2,2) & 7 & 1282.085 & 1.367782 \\
\hline $\operatorname{PID}(1,1) \operatorname{PID}(1,2) \operatorname{PID}(2,2)$ & 9 & 1281.751 & 1.367426 \\
\hline \multicolumn{4}{|l|}{ Full Interactive Restricted Structure } \\
\hline $\mathrm{PI}(1,1) \mathrm{PI}(1,2) \mathrm{PI}(2,1) \mathrm{PI}(2,2)$ & 8 & 1110.798 & 1.185046 \\
\hline PI(1,1) PI(1,2) PI(2,1) PID(2,2) & 9 & 1110.797 & 1.185045 \\
\hline PID(1,1) PID(1,2) PID(2,1) PID(2,2) & 12 & 1110.787 & 1.185034 \\
\hline \multicolumn{4}{|l|}{ Full Optimal Structure } \\
\hline $\mathrm{K}_{\mathrm{LQG}}(1,1) \mathrm{K}_{\mathrm{LQG}}(1,2) \mathrm{K}_{\mathrm{LQG}}(2,1) \mathrm{K}_{\mathrm{LQG}}(2,2)$ & 47 & 937.346 & 1 \\
\hline
\end{tabular}

